\title{
Lymphocyte Immune Response and T Cell Differentiation in Fontan Patients with protein-losing enteropathy
}

\author{
Julia Moosmann ${ }^{10}$ \\ Okan Toka ${ }^{2}$ Sören Lukassen ${ }^{3}$ \\ Sven Dittrich ${ }^{1, a}$ (1) \\ ${ }^{1}$ Department of Pediatric Cardiology, Friedrich-Alexander Universität \\ Erlangen-Nürnberg (FAU), Erlangen, Germany \\ 2 Pediatric and Adolescent Clinic, Fürth, Germany \\ 3 Institute of Human Genetics, Friedrich-Alexander Universität \\ Erlangen-Nürnberg (FAU), Erlangen, Germany \\ ${ }^{4}$ Department of Internal Medicine 5, Haematology and Oncology, \\ Universität Erlangen-Nürnberg (FAU), Erlangen, Germany \\ Thorac Cardiovasc Surg 2021;69:e10-e20.
}

Arif B. Ekici ${ }^{3}$

Andreas Mackensen ${ }^{4}$

Simon Völk| $\left.\right|^{4, a}$

\begin{abstract}
Address for correspondence Julia Moosmann, MD, Department of Pediatric Cardiology, Friedrich-Alexander Universität ErlangenNürnberg (FAU), Loschgestraße 15, 91054 Erlangen, Germany (e-mail: julia.moosmann@uk-erlangen.de).
\end{abstract}

\begin{abstract}
Background Protein-losing enteropathy (PLE) is a severe complication of the Fontan circulation. There is increasing discussion about whether lymphatic dysregulation is involved as pathomechanism of PLE. This investigation focuses on the interplay between alteration of lymphatic cells and immunologic pathway alterations.

Methods Micro-ribonucleic acid (miRNA) expression profiling was performed in 49 patients ( $n=10$ Fontan patients with PLE, $n=30$ Fontan patients without PLE, and $n=9$ patients with dextro-transposition of the great arteries (dTGA). miRNA pathway analysis was performed to identify significantly enriched pathways. To determine lymphocyte populations and subtypes multiparameter flow cytometry was used.

Results miRNAs pathway analysis of Fontan patients with PLE revealed 20 significantly changed networks of which four of the ten largest were associated with immunologic processes. This finding is supported by significant $T$ cell deficiency with decreased CD4+ count $(p=0.0002)$, altered CD4 $+/ C D 8+$ ratio, and significantly modified CD4+ $(p<0.0001)$ and CD8 $+(p=0.0002)$ T cell differentiation toward effector and terminal

\section{Keywords}

- congenital heart disease (CHD)

- inflammation

- systemic

- genetics

- genomics differentiated T cells in Fontan patients with PLE. Analyses of CD4+ T cell subsets demonstrated significantly increased frequencies of CD4+CD25+CD127-regulatory T cells (Treg) in Fontan patients with PLE $(p=0.0011)$.

Conclusion PLE in Fontan patients is associated with severe lymphopenia, $T$ cell deficiency, significant alterations of $T$ cell differentiation, and increased Treg frequency reflecting an immune status of chronic inflammation and shortened protection against pathogens and autoimmunity. These cellular alterations seemed to be dysregulated by several miRNA controlled immunological pathways.
\end{abstract}

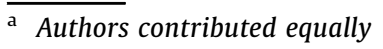

received

July 5,2020

accepted after revision

November 24, 2020
DOI https://doi.org/ $10.1055 / \mathrm{s}-0041-1723781$ ISSN 0171-6425.

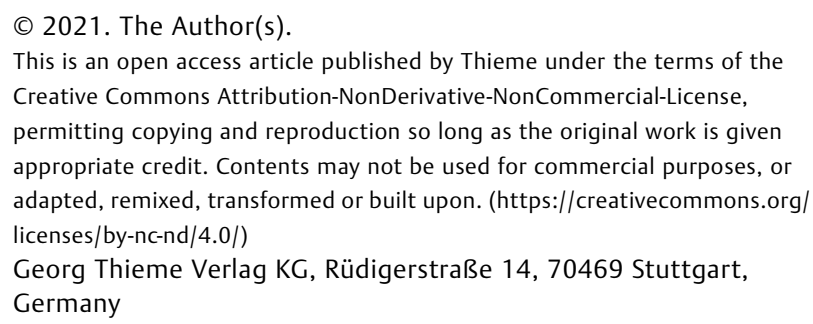




\section{Introduction}

The Fontan procedure is a well-established palliative procedure for patients with univentricular heart malformations. Cardiovascular surgical techniques and postoperative care constantly improved over time, resulting in increased longterm survival rates of up to $85 \% 30$ years after Fontan surgery. ${ }^{1}$ However, the Fontan circulation is characterized by elevated central venous pressure, a nonpulsatile pulmonary blood flow, and reduced cardiac output leading to multiple organ system dysfunctions. Approximately 3 to $15 \%$ of all Fontan patients develop protein-losing enteropathy (PLE), which represents a serious complication and is associated with increased mortality. $^{2}$ The pathophysiology of PLE in Fontan patients is multifactorial. Recent investigations suggest that lymphatic circuit abnormalities and lymphatic congestion may be involved in the development of PLE. ${ }^{3,4}$ The lymphatic system plays a central role in the immune system. It consists of a network of lymph nodes and lymphatic vessels that transports the lymph fluid, including white blood cells, throughout the body. Besides abnormal lymphatic perfusion pattern, which thanks to the latest imaging technique have received special attention in the scientific debate, immune abnormalities including lymphopenia and $\mathrm{T}$ cell deficiency have long been known to occur in Fontan patients with PLE. ${ }^{5}$

The interaction of the lymphatic circulation and cellular lymphatic dysfunction in the progress of PLE needs further understanding.

Micro-ribonucleic acids (miRNAs) are small noncoding RNA molecules of $\sim 21$ to 25 nucleotides and represent modulators of the post-transcriptional gene expression. ${ }^{6}$ miRNAs are involved in various biological processes like cell differentiation, cell cycle control, cell growth, and functionality. ${ }^{7}$ In the last years, they emerged as new biomarkers for various diseases and were identified to be involved in disease development. ${ }^{8}$ A single miRNA downregulates hundreds of target mRNAs; thus, even small changes in expression levels of single miRNAs can affect a wide range of signaling pathways involved in diverse biological functions. in silico miRNA expression profiling facilitates highlighting complex clinical pathways of disease development. This can be used to generate new hypotheses about relevant disease pathways controlled by miRNA signatures. ${ }^{9}$

Focus of this study is to analyze lymphocyte populations and subtypes and miRNA expression profiling to highlight the interplay between lymphatic circulation and cellular lymphatic dysfunction in Fontan patients with PLE.

\section{Materials and Methods}

\section{Patients}

We reviewed our in-house database for patients who have undergone successful Fontan operation at the Department of Pediatric Cardiology, University Hospital of Erlangen between 1990 and 2014. We identified 72 Fontan patients without PLE and 10 patients with PLE. All patients were contacted and invited to participate in the study. 30 Fontan patients without PLE and 10 patients with PLE followed our invitation to participate in the study. To avoid any bias on lymphopenia and T cell count due to neonatal thymectomy, 9 patients with dextro-transposition of the great arteries (dTGA) were included to compare the results to a biventricular group. ${ }^{10}$ All measurements were performed in individuals without current infection or fever and at least 6 months after the last surgical intervention or vaccination. Patients with immune system defects were excluded from the study.

PLE was diagnosed as a clinical syndrome, defined by at least three of the following six symptoms: (1) mild-to-profound diarrhea, (2) elevated fecal $\alpha-1$ AT $>400 \mu \mathrm{g} / \mathrm{g}$, (3) serum total protein $<50 \mathrm{~g} / \mathrm{L}$, (4) hypoalbuminemia $<30 \mathrm{~g} / \mathrm{L}$, and (5) hyponatremia $<135 \mathrm{mmol} / \mathrm{L}$ and (6) clinical third space volume retention (e.g., ascites, edema and/or pleural effusions). ${ }^{11}$

\section{Sample Collection and RNA Extraction}

Peripheral whole blood samples $(2.5 \mathrm{~mL})$ were collected in PAXgene blood RNA tubes (PreAnalytix, Hombrechtikon, Switzerland). miRNA was extracted from peripheral whole blood using the PAXgene Blood miRNA kit (PreAnalytix) and automated QiaCube (Qiagen, Hilden, Germany) protocol. RNA concentration was quantified by NanoDrop 1000 (Nanodrop Technologies; Thermo Fisher Scientific, Waltham, Massachusetts, United States). RNA Integrity Number scores had a mean of $7.0 \pm 2.9$, thus showing good RNA quality.

\section{Differential Expression Analysis of miRNA Using Microarrays}

The miRNA microarray expression analysis was performed on GeneChip miRNA 4.1 microarray (Affymetrix, Santa Clara, California, United States) according to the manufacturer's instructions. The array contained sequence-specific probes for 2,578 human mature miRNAs and 2,025 human pre-miRNAs listed in miRBase v20.0 (http://www.mirbase. org). Data are available under the GSO accession number: GSE130647. Data were analyzed with Partek Genomics Suite software v6.6 (Partek, St Louis, Missouri, United States). To identify differentially expressed miRNAs between the defined sample groups, analysis of variance tests were applied.

\section{Pathway Analyses}

GO-term enrichment analyses were performed using miRNA-target protein assignments from miRTarBase version 6.0. ${ }^{12}$ Common methods to identify significant pathways are largely biased by highly-related biological processes. In this study, we applied a modified strategy as recommended by Godard and van Eyll for our in-house analysis. ${ }^{13}$

GO-terms were displayed as lists of miRNAs targeting their associated proteins. Enrichment statistics were calculated using the hypergeometric test on overlaps of differentially regulated miRNAs and GO-terms. The Benjamini-Hochberg FDR method was used to correct for multiple testing. Results were visualized using Cytoscape 3.5.0 illustrating the relationship between the different gene ontology terms. Any nodes (terms) not significantly enriched or connected to at least one significantly enriched node were removed, which created interconnected subnetworks of different sizes. Individual steps of pathway analysis are illustrated in - Supplementary Figure S1. 
e12 Lymphocyte Immune Response and T Cell Differentiation in Fontan Patients with PLE Moosmann et al.

\section{Flow Cytometry and Routine Laboratory}

Samples were stained with the following fluorochromeconjugated antibodies: anti-CD3 (SK7), anti-CD4 (RPA-T4), anti-CD8 (SK1), anti-CD14 (MOP9), anti-CD16 (3G8), antiCD19 (HIB19), anti-CD45 (2D1), anti-CD45RO (UCHL-1), anti-CD56 (NCAM16.2), and anti-CCR7 (150503, all from BD Biosciences, Heidelberg, Germany). Data acquisition was performed on a FACS Canto II (BD Biosciences). Data were analyzed with Kaluza software (Beckman Coulter).

Routine laboratory parameters were analyzed by standard laboratory techniques. Total blood counts were determined using a Sysmex XE-2100 (Sysmex Europe, Norderstedt, Germany).

\section{Statistics}

Statistical analysis was performed using SPSS and GraphPad Prism version 6.0.5. Continuous variables are expressed as mean \pm standard deviation or median and range when appropriate. For patient characteristics, the arithmetical mean was chosen above the median, as outliers had been excluded. Normality was analyzed by Shapiro-Wilk-test and QQ-plots. Differences in expression patterns were tested using unpaired, nonparametric Mann-Whitney-U test. A $p$-value $<0.05$ was considered as significant.

For miRNA pathway analysis, $p$-value calculations based on the assumption of a hypergeometric distribution were performed using R. Correction for multiple testing using the Benjamini-Hochberg method was then applied to the resulting values.

\section{Ethics Statement}

The study has been approved by the Ethics Committee of the University of Erlangen. Written informed consent was obtained from all participating individuals.

\section{Results}

\section{Study Population}

Cardiac malformations are given in - Table 1. Patient characteristics, laboratory parameters, and current medication are shown in $\mathbf{- T a b l e s} \mathbf{2}$ and $\mathbf{3}$. PLE was diagnosed according to the criteria described above.

\section{miRNA Expression Differences in Fontan Patients}

miRNA expression profiling was performed in 49 patients, in three Fontan patients without PLE, no array could be performed due to poor RNA quality. Microarray expression differences between all three groups are illustrated in the principal component analysis; clearly distinguishing clusters between dTGA and Fontan patients with PLE, while Fontan patients without PLE showed overlap with both groups ( - Fig. 1).

Comparing Fontan with and without PLE patients, 21 miRNAs (2 upregulated and 19 downregulated) were significantly differentially expressed. Fontan with PLE compared with dTGA differed in the expression of 15 miRNAs, all downregulated. Between Fontan and dTGA samples, one miRNA (upregulated) was significantly differentially expressed (-Fig. 2).
Table 1 Cardiac anatomy of Fontan patients

\begin{tabular}{|c|c|c|}
\hline Cardiac anatomy & $\begin{array}{l}\text { Fontan } \\
\text { with PLE } \\
(n=10)\end{array}$ & $\begin{array}{l}\text { Fontan } \\
\text { without PLE } \\
(n=30)\end{array}$ \\
\hline Age at Fontan surgery (years) & $3.9 \pm 2.5$ & $3.5 \pm 2.4$ \\
\hline \multicolumn{3}{|l|}{ Systemic ventricle } \\
\hline Left & $n=5$ & $n=20$ \\
\hline Right & $n=5$ & $n=10$ \\
\hline Tricuspid atresia & $n=2$ & $n=8$ \\
\hline $\begin{array}{l}\text { Pulmonary atresia with intact } \\
\text { ventricular septum (PA-IVS) }\end{array}$ & $n=1$ & $n=2$ \\
\hline Unbalanced atrioventricular canal & $n=1$ & $n=2$ \\
\hline Hypoplastic left heart syndrome & $n=3$ & $n=1$ \\
\hline $\begin{array}{l}\text { Interrupted aortic arch, } \\
\text { hypoplastic left ventricle }\end{array}$ & $n=1$ & $n=2$ \\
\hline Single ventricle & $n=1$ & $n=7$ \\
\hline Double inlet left ventricle & & $n=4$ \\
\hline $\begin{array}{l}\text { Double outlet right ventricle, } \\
\text { mitral atresia }\end{array}$ & & $n=2$ \\
\hline $\begin{array}{l}\text { Double outlet right ventricle, } \\
\text { criss-cross heart }\end{array}$ & & $n=1$ \\
\hline cCTGA & $n=1$ & $n=1$ \\
\hline Fenestration & $n=3$ & $n=2$ \\
\hline \multicolumn{3}{|l|}{ Cardiac interventions } \\
\hline Left pulmonary artery dilatation & $n=5$ & $n=4$ \\
\hline $\begin{array}{l}\text { Extracardiac conduit dilatation/ } \\
\text { stent implantation }\end{array}$ & $n=2$ & $n=2$ \\
\hline Closure of veno-venous collaterals & $n=2$ & $n=7$ \\
\hline $\begin{array}{l}\text { Dilatation/Stent implantation } \\
\text { innominate vein }\end{array}$ & $n=2$ & \\
\hline $\begin{array}{l}\text { Closure of major aorto-pulmonary } \\
\text { collateral arteries/systemic-to- } \\
\text { pulmonary collateral arteries }\end{array}$ & $n=2$ & $n=5$ \\
\hline Closure of fenestration & & $n=1$ \\
\hline Electrophysiological examination & & $n=2$ \\
\hline $\begin{array}{l}\text { Other (closure persistent left } \\
\text { superior vena cava) }\end{array}$ & & $n=1$ \\
\hline \multicolumn{3}{|l|}{ AV-regurgitation } \\
\hline $1^{\circ}$ & $n=4$ & $n=12$ \\
\hline $11^{\circ}$ & $n=1$ & $n=4$ \\
\hline \multicolumn{3}{|l|}{ Cardiac rhythm } \\
\hline Sinus rhythm & $n=8$ & $n=26$ \\
\hline Pacemaker & $n=1$ & $n=1$ \\
\hline $\begin{array}{l}\text { Other (e.g., atrial rhythm, } \\
\text { AV-block I) }\end{array}$ & $n=1$ & $n=3$ \\
\hline
\end{tabular}

Abbreviations: AV, aortic valve; PLE, protein-losing enteropathy; SD, standard deviation.

Note: Age at Fontan surgery is expressed as mean \pm SD.

\section{Pathway Alterations in Immune Processes of Fontan Patients with PLE}

Go-term pathway analysis revealed 1,896 pathways to be significantly enriched for miRNAs upregulated in Fontan 
Table 2 Patients characteristics

\begin{tabular}{|c|c|c|c|c|c|c|}
\hline & \multirow{2}{*}{$\begin{array}{l}(1) \\
\text { dTGA } \\
n=9\end{array}$} & \multirow{2}{*}{$\begin{array}{l}(2) \\
\text { Fontan } \\
n=30\end{array}$} & \multirow{2}{*}{$\begin{array}{l}(3) \\
\text { Fontan with PLE } \\
n=10\end{array}$} & \multicolumn{3}{|l|}{ p-Value } \\
\hline & & & & 1 versus 2 & 1 versus 3 & 2 versus 3 \\
\hline $\begin{array}{l}\text { Male } \\
\text { Female }\end{array}$ & $\begin{array}{l}7 \\
2\end{array}$ & $\begin{array}{l}18 \\
12\end{array}$ & $\begin{array}{l}5 \\
5\end{array}$ & & & \\
\hline Age at measurement (years) & $16.7 \pm 5.1$ & $14.5 \pm 6.8$ & $14.3 \pm 7.8$ & 0.4019 & 0.4002 & 0.7939 \\
\hline Time after last surgery (years) & $14.3 \pm 8.5$ & $8.4 \pm 6.4$ & $6.7 \pm 4.2$ & 0.1004 & 0.0770 & 0.6636 \\
\hline Age at thymectomy (days) & $40.1 \pm 80$ & $188 \pm 365$ & $177 \pm 291$ & 0.0576 & 0.7646 & 0.4363 \\
\hline Weight at measurement $(\mathrm{kg})$ & $70.9 \pm 44.5$ & $49.8 \pm 25.1$ & $33.1 \pm 14.5$ & 0.1894 & 0.0027 & 0.0590 \\
\hline $\begin{array}{l}\mathrm{BMI} \\
\left(\mathrm{kg} / \mathrm{m}^{2}\right)\end{array}$ & $20.9 \pm 2.4$ & $19.0 \pm 3.5$ & $17.2 \pm 2.1$ & 0.1592 & 0.0030 & 0.1957 \\
\hline $\begin{array}{l}\text { Albumin } \\
(\mathrm{g} / \mathrm{L})\end{array}$ & $44.2 \pm 1.9$ & $43.0 \pm 2.7$ & $28.8 \pm 8.2$ & 0.3273 & $<0.0001$ & $<0.0001$ \\
\hline Osmolality $(\mathrm{mOsm} / \mathrm{kg})$ & $284.4 \pm 2.1$ & $282.1 \pm 3.6$ & $279.1 \pm 3.9$ & 0.0472 & 0.0027 & 0.0257 \\
\hline Total protein $(\mathrm{g} / \mathrm{L})$ & $67.2 \pm 4.2$ & $66.7 \pm 4.4$ & $48.3 \pm 12.2$ & 0.4533 & 0.0003 & $<0.0001$ \\
\hline $\lg G(g / L)$ & $9.3 \pm 2.6$ & $9.4 \pm 2.7$ & $3.7 \pm 3.7$ & $>0.9999$ & 0.0030 & 0.0001 \\
\hline Serum sodium $(\mathrm{mmol} / \mathrm{L})$ & $137.5 \pm 1.0$ & $136.2 \pm 1.8$ & $134.4 \pm 2.0$ & 0.0133 & 0.0011 & 0.0111 \\
\hline Alpha-1 antitrypsin $(\mu \mathrm{g} / \mathrm{g})$ & $238.3 \pm 150.5$ & $247.9 \pm 321.7$ & $1424.5 \pm 640.5$ & 0.5936 & 0.0005 & $<0.0001$ \\
\hline
\end{tabular}

Abbreviations: BMI, body mass index; dTGA, dextro-transposition of the great arteries; IgG, immunoglobulin G; PLE, protein-losing enteropathy; SD, standard deviation.

Note: Values are expressed as mean \pm SD. Differences were calculated using Mann-Whitney U test.

patients with PLE versus Fontan patients without PLE at a $p$ value of less than 0.05 . For downregulated miRNAs, there were only three enriched pathways. Filtering left us with 20 subnetworks consisting of at least 5 interconnected terms each, representing larger-scale biological processes affected by miRNA dysregulation (-Table 4 ). Five of the ten largest subnetworks connected to immune system processes: (1) immune response, (5) differentiation, (6) immune system, (7) immune system differentiation, and (8) apoptosis. The other networks describe (2) catabolic process, (3) biosynthetic process, (4) nutrient response, (9) hormones, and (10) bone/digestive development. An example of the biggest GO-term network "immune response" is given in the -Supplementary Fig. 2.

\section{PLE in Fontan Patients Is Associated with T Cell Deficiency}

Numbers of leukocytes $(p=0.0028)$ and monocytes $(p=0.0346)$ were significantly increased in Fontan patients with PLE compared with Fontan patients without PLE (-Supplementary Table S1). Comparisons of absolute numbers of neutrophils showed a median 1.8- and 1.5-fold difference between Fontan patients $(p=0.0002)$ and dTGA patients $(p=0.0118)$ and PLE patients. Eosinophils showed no difference between all groups (-Supplementary Table S1). Patients with PLE showed significant lymphopenia with reduction in both absolute numbers (800 cells/ $\mu \mathrm{L}[500-3,500])$ and frequency of lymphocytes compared with Fontan without PLE $(1,300$ cells $/ \mu \mathrm{L}$ $[600-3,000] ; p=0.0092)$ and dTGA group $(1,800$ cells $/ \mu \mathrm{L}$ [1,300-2,600]; $p=0.0061$ ).

We observed a significant reduction in lymphocytes in Fontan patients without PLE compared with dTGA patients $(p=0.0016)$. No differences of NK cells (PLE: 211 cells $/ \mu \mathrm{L}$

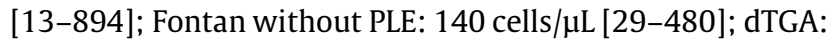
271 cells/ $\mu \mathrm{L}$ [20-441]) and B cells (PLE: 322 cells/ $\mu L$; Fontan: 291 cells/ $\mu \mathrm{L}$; dTGA: 345 cells $/ \mu \mathrm{L}$ ) between the groups were observed (-Fig. 3). There was no difference in $\mathrm{T}$ cell numbers between Fontan and dTGA patients $(p=0.0742)$. PLE patients showed a significant decrease in T cells (236 cells/ $\mu \mathrm{L}$ [75-943]) compared with Fontan patients without PLE (618 cells/ $\mu \mathrm{L}$ [104-1170]; $p=0.0024)$ and dTGA patients $(1,121$ cells/ $\mu \mathrm{L}[218-1,769] ; p=0.0021)$.

\section{Fontan Patients with PLE Show Significant Reduction in CD4+ T Cells}

CD4+ T cells were significantly reduced in PLE patients (94 cells/ $\mu \mathrm{L}$ [52-351]) compared with Fontan patients without PLE (457 cells/ $\mu \mathrm{L}$ [81-857]; $p=0.0002$ ) and dTGA patients (710 cells/ $\mu \mathrm{L}$ [142-1,075]; $p=0.0006$; - Fig. 4A). Nine of ten PLE patients showed a CD $4+T$ cell count $<200$ cells/ $/ \mathrm{L}$. Only $5 / 30$ Fontan and 1/10 dTGA patients exhibited a CD4+ count below 200 cells $/ \mu \mathrm{L}$. The absolute cell count of CD8+ T cells was reduced in PLE patients ( 98 cells/ $\mu \mathrm{L}$ [17-576]) compared with dTGA patients (328 cells/ $\mu \mathrm{L}[70-740 ; p=0.035$ ) but not to Fontan patients without PLE (149 cells/ $\mu \mathrm{L}$ [20-394]; $p=0.1524 ;$ - Fig. 4B). Significant lower CD4+ cell count leads to a change in CD4+/CD8+ ratio shifted to $1: 1$. PLE patients showed a twofold increase in the frequency of CD4+ CD25+ CD127- regulatory T cells (Treg) (14\% [6.7-22] of CD4+ T cells, -Fig. 4C) compared with Fontan patients without PLE (6.9\% [5.1-13.3]; $p=0.0011)$ and dTGA patients (6.9\% [6.4-8.6]; $p=0.0028)$. The reduction in absolute CD4 $+\mathrm{T}$ cell count of PLE patients resulted in a significant decrease in Treg cells $/ \mu \mathrm{L}$

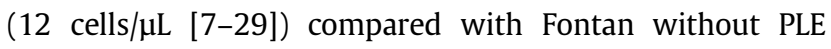


e14 Lymphocyte Immune Response and T Cell Differentiation in Fontan Patients with PLE Moosmann et al.

Table 3 Current medication

\begin{tabular}{|l|l|l|l|}
\hline & $\begin{array}{l}\text { Fontan } \\
\text { with PLE }\end{array}$ & $\begin{array}{l}\text { Fontan } \\
\text { without PLE }\end{array}$ & dTGA \\
\hline Diuretic therapy & $10 / 10$ & $2 / 30$ & $0 / 9$ \\
\hline Furosemide & $n=9$ & $n=2$ & \\
\hline Eplerenone & $n=5$ & $n=1$ & \\
\hline Hydrochlorothiazide & $n=4$ & $n=1$ & \\
\hline Spironolactone & $n=4$ & $n=1$ & \\
\hline Tolvaptan & $n=3$ & & \\
\hline ACE-inhibitors & $2 / 10$ & $2 / 30$ & $0 / 9$ \\
\hline Captopril & $n=2$ & $n=1$ & \\
\hline Enalapril & & $n=1$ & \\
\hline Anticoagulation & $10 / 10$ & $30 / 30$ & $0 / 9$ \\
\hline ASS & $n=2$ & $n=26$ & \\
\hline Phenprocoumon & $n=6$ & $n=3$ & \\
\hline Clopidogrel & $n=1$ & $n=3$ & \\
\hline Warfarin & $n=1$ & & $0 / 9$ \\
\hline Dipyridamole & $n=1$ & $n=2$ & \\
\hline Betablocker & $1 / 7$ & $4 / 30$ & \\
\hline Metoprolol & $n=1$ & $n=2$ & \\
\hline Carvedilol & & $n=2$ & \\
\hline Steroids & $1 / 10$ & $0 / 30$ & \\
\hline Thyroid hormones & $5 / 10$ & $3 / 30$ & \\
\hline Pulmonary & $2 / 10$ & $1 / 30$ & \\
\hline vasodilators & $n=2$ & $n=1$ & \\
\hline Bosentan & & $n=1$ & \\
\hline Digoxin & $n / 30$ & \\
\hline Abrenafil & $n$ & \\
\hline
\end{tabular}

Abbreviations: ACE, angiotensin-converting enzyme; ASS, acetylsalicylic acid; dTGA, dextro-transposition of the great arteries; PLE, proteinlosing enteropathy.

(30 cells $/ \mu \mathrm{L}[6-63$ ]; $p=0.0011$ ) and dTGA patients ( 45 cells $/ \mu \mathrm{L}$ [9-77]; $p=0.0076$; - Fig. 4D). No significant changes in Treg cell frequency or number were found between Fontan without PLE and dTGA patients. Routine laboratory analysis of albumin and total protein showed significant correlations with $\mathrm{T}$ cell count, CD4+ T cell count and Treg frequency (-Fig. 5)

\section{Analysis of $\mathbf{T}$ Cell Subsets Identified Alterations in Differentiation and Loss of Naïve T Cells}

The differentiation pattern of the CD4+ T cell compartment of Fontan patients with PLE showed a shifting toward memory $\mathrm{T}$ cells with nearly complete loss of naïve CD4 $+\mathrm{T}$ cells ( - Fig. 6A and B). We observed a shift from the naïve CD8+ $\mathrm{T}$ cell pool to memory and terminal differentiated $\mathrm{T}$ cells re-expressing CD45RA (TEMRA) in Fontan patients with PLE ( - Fig. 6A and C). T cell differentiation pattern of Fontan without PLE and dTGA patients showed no differences. We found a decrease in all differentiation stages of the CD4+ T cell compartment in PLE patients ( - Fig. $\mathbf{6 A}$ and $\mathbf{B}$ ). Fontan patients with PLE showed a loss of naïve CD4 + T cells ( 7 cells $/ \mu \mathrm{L}[2-25]$ )

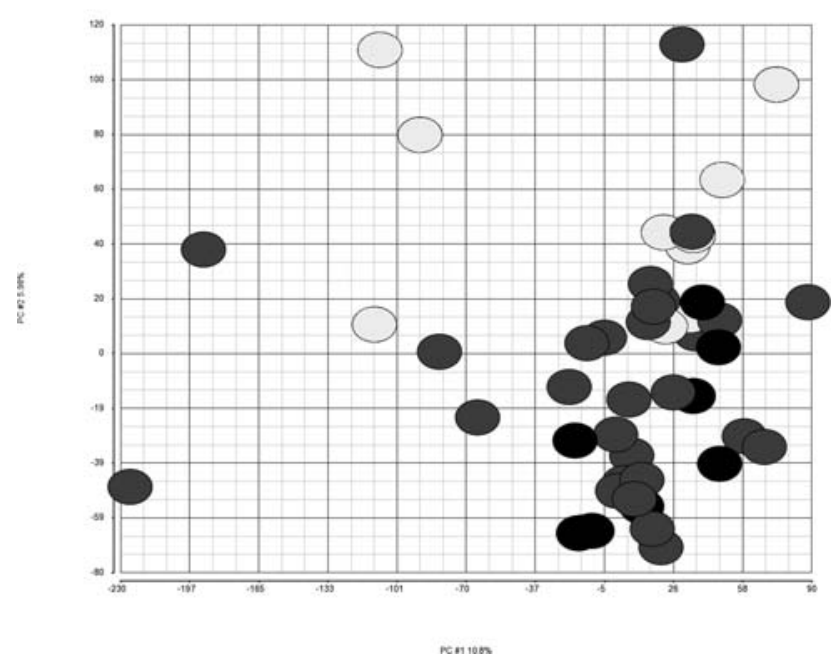

Fig. 1 Principal component analysis (PCA). The micro-ribonucleic acid (miRNA) microarray expression data were used to construct a three-dimensional PCA. dextro-transposition of the great arteries (dTGA) (light grey), Fontan patients without protein-losing enteropathy (PLE) (dark grey) and Fontan with PLE (black).

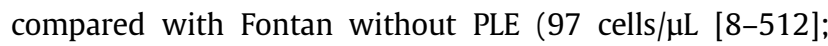

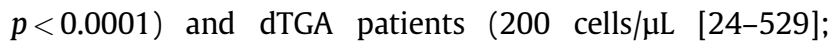
$p<0.0001$ ). Absolute numbers of both CD4+ memory T cells and CD4+ TEMRA cells were decreased in Fontan patients with PLE (memory: 86 cells/ $\mu \mathrm{L}$ [23-309]; TEMRA: 3 cells/ $\mu \mathrm{L}$ [1-15]) compared with Fontan without PLE (memory: 300 cells $/ \mu \mathrm{L}$ [35-610], $p=0.0026$; TEMRA: 7 cells $/ \mu \mathrm{L}[2-31] ; p=0.0053$ ) and dTGA patients (memory: 423 cells/ $\mu \mathrm{L}$ [58-683]; $p=0.0041$; TEMRA: 14 cells/ $\mu \mathrm{L}$ [3-27]; $p=0.0069$ ). We did not find significant differences of $\mathrm{CD} 4+$ naïve, memory, or TEMRA cell numbers in Fontan patients without PLE and dTGA patients. Phenotypic analysis of the CD8+ T cell compartment revealed a loss of naïve cells (10 cells/ $\mu \mathrm{L}[2-46]$ ) in Fontan patients with PLE compared wth Fontan without PLE (40 cells/ $\mu \mathrm{L}$ [10-142]; $p=0.0002$ ) and dTGA patients (137 cells $/ \mu \mathrm{L}[21-$ 430]; $p=0.0005 ;-$ Fig. 6 A and C). A significant reduction in naïve CD8+ T cells in Fontan patients without PLE compared with dTGA patients ( $p=0.0116)$ was observed. In contrast to the CD4+ T cell compartment, memory cells (Fontan with PLE:

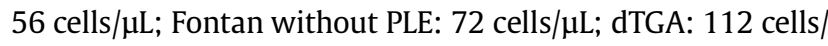
$\mu \mathrm{L}$ ) and TEMRA CD8 + T cells (Fontan with PLE: 24 cells $/ \mu \mathrm{L}$; Fontan without PLE: 19 cells $/ \mu \mathrm{L} ; 25$ dTGA: cells $/ \mu \mathrm{L}$ ) were not reduced in Fontan patients with PLE.

\section{Discussion}

Lymphatic dysregulation has evolved as a new potential pathomechanism contributing to complications of the Fontan circulation. ${ }^{3,14}$ Since lymphatic circulation plays a crucial role in the immune system, the question arises to what extent the immunological changes observed in Fontan patients are involved in the development of PLE. The interaction between lymphatic circulation and cellular lymphatic dysfunction in the progress of PLE development needs further understanding. We identified significantly modified $\mathrm{T}$ cell 


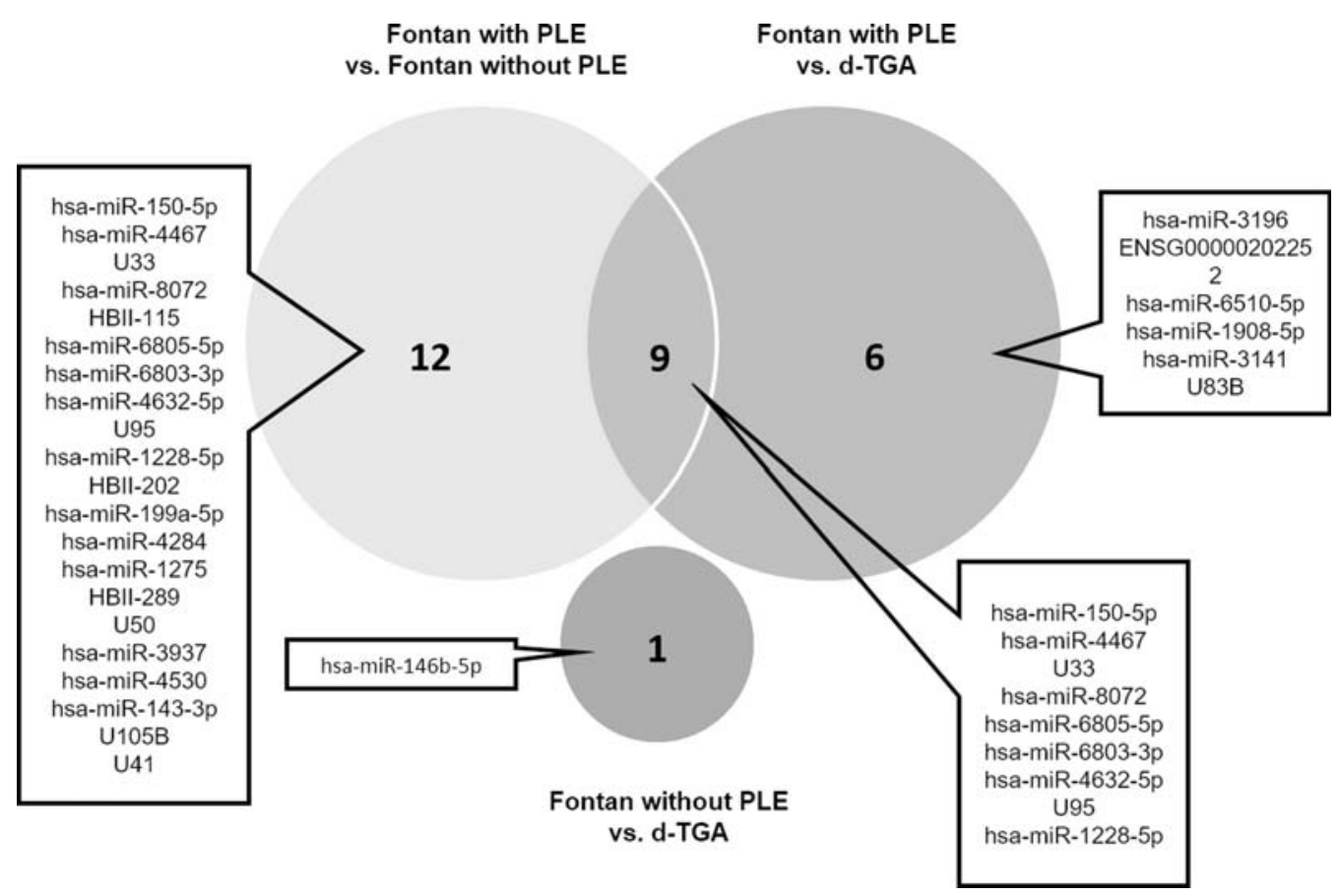

Fig. 2 Venn diagram of significantly regulated micro-ribonucleic acids (miRNAs). Fontan with protein-losing enteropathy (PLE) versus Fontan without PLE; Fontan with PLE versus dextro-transposition of the great arteries (dTGA); Fontan without PLE versus dTCA. Respective miRNAs are shown.

Table 4 Top 10 networks of pathway analysis. Significant networks are demonstrated including number of significant GO terms.

\begin{tabular}{|c|c|c|c|c|c|}
\hline Enrichment & $\begin{array}{l}\text { Expected } \\
\text { overlap }\end{array}$ & GO ID & Pathway & $q$-Value & $p$-Value \\
\hline \multicolumn{6}{|c|}{ 1. Immune response ( $n=38 \mathrm{GO}$-terms) } \\
\hline 1.18232925 & 83.7330211 & GO:0050869 & $\begin{array}{l}\text { GO_POSITIVE_REGULATION_OF_CELL_ } \\
\text { ACTIVATION }\end{array}$ & 0.00681714 & $2.80 \mathrm{E}-04$ \\
\hline 1.25387667 & 60.6120219 & GO:0002704 & $\begin{array}{l}\text { GO_REGULATION_OF_LEUKOCYTE_ } \\
\text { MEDIATED_IMMUNITY }\end{array}$ & 0.01367208 & 0.00127053 \\
\hline 1.30162718 & 55.3153786 & GO:0002444 & GO_LEUKOCYTE_MEDIATED_IMMUNITY & 0.00919742 & $5.80 \mathrm{E}-04$ \\
\hline 1.40454109 & 51.2622951 & GO:0002622 & GO_PLATELET_DEGRANULATION & 0.00306798 & $2.90 \mathrm{E}-05$ \\
\hline 1.40986133 & 49.6502732 & GO:0050864 & GO_REGULATION_OF_B_CELL_ACTIVATION & 0.00315513 & 3.89E-05 \\
\hline \multicolumn{6}{|c|}{ 2. Catabolic process ( $n=34$ GO-terms) } \\
\hline 1.07173458 & 108.235753 & GO:0009894 & GO_REGULATION_OF_CATABOLIC_PROCESS & 0.00768437 & $3.56 \mathrm{E}-04$ \\
\hline 1.07219083 & 70.882904 & GO:0009896 & $\begin{array}{l}\text { GO_NEGATIVE_REGULATION_OF_ } \\
\text { CATABOLIC_PROCESS }\end{array}$ & 0.19413426 & 0.13958624 \\
\hline 1.26380375 & 70.4223263 & GO:0043456 & $\begin{array}{l}\text { GO_NEGATIVE_REGULATION_OF_ } \\
\text { MAPK_CASCADE }\end{array}$ & 0.00411444 & $7.98 \mathrm{E}-05$ \\
\hline 1.30892928 & 61.1186573 & GO:0010921 & $\begin{array}{l}\text { GO_REGULATION_OF_PHOSPHATASE_ } \\
\text { ACTIVITY }\end{array}$ & 0.00441363 & $1.08 \mathrm{E}-04$ \\
\hline 1.29959284 & 57.7103825 & GO:0043468 & $\begin{array}{l}\text { GO_REGULATION_OF_GENERATION_OF_- } \\
\text { PRECURSOR_METABOLITES_AND_ENERGYY }\end{array}$ & 0.00792375 & $3.75 \mathrm{E}-04$ \\
\hline \multicolumn{6}{|c|}{ 3. Biosynthetic process ( $n=27$ GO-terms) } \\
\hline 1.06210437 & 97.9188134 & GO:0009963 & $\begin{array}{l}\text { GO_POSITIVE_REGULATION_OF_ } \\
\text { CATABOLIC_PROCESS }\end{array}$ & 0.09135647 & 0.04422164 \\
\hline 1.16829958 & 86.4504294 & GO:0004857 & GO_ENZYME_INHIBITOR_ACTIVITY & 0.00767124 & $3.42 \mathrm{E}-04$ \\
\hline 1.28506618 & 52.1373927 & GO:0010521 & $\begin{array}{l}\text { GO_POSITIVE_REGULATION_OF_ } \\
\text { AUTOPHAGY }\end{array}$ & 0.01593233 & 0.00183715 \\
\hline
\end{tabular}


e16 Lymphocyte Immune Response and T Cell Differentiation in Fontan Patients with PLE Moosmann et al.

Table 4 (Continued)

\begin{tabular}{|c|c|c|c|c|c|}
\hline Enrichment & $\begin{array}{l}\text { Expected } \\
\text { overlap }\end{array}$ & GO ID & Pathway & $q$-Value & p-Value \\
\hline 1.40464927 & 49.8345043 & GO:2000379 & $\begin{array}{l}\text { GO_POSITIVE_REGULATION_OF_REACTIVE_ } \\
\text { OXYGEN_SPECIES_METABOLIC_PROCESS }\end{array}$ & 0.00336853 & 4.51E-05 \\
\hline 1.39868304 & 46.4722873 & GO:0019212 & GO_KINASE_INHIBITOR_ACTIVITY & 0.00483154 & $1.45 \mathrm{E}-04$ \\
\hline \multicolumn{6}{|c|}{ 4. Nutrient response ( $n=25$ GO-terms) } \\
\hline 1.04071821 & 104.735363 & GO:0002542 & GO_IMMUNE_SYSTEM_DEVELOPMENT & 0.12329052 & 0.071123 \\
\hline 1.0604216 & 93.3590945 & GO:0002258 & GO_ACTIVATION_OF_IMMUNE_RESPONSE & 0.12606365 & 0.07368365 \\
\hline 1.20047764 & 77.4691647 & GO:0032103 & $\begin{array}{l}\text { GO_POSITIVE_REGULATION_OF_- } \\
\text { RESPONSE_TO_EXTERNAL_STIMULUS }\end{array}$ & 0.0084118 & $4.84 \mathrm{E}-04$ \\
\hline 1.0432127 & 76.6861827 & GO:0050921 & GO_LEUKOCYTE_MIGRATION & 0.27695604 & 0.22678415 \\
\hline 1.28745316 & 62.9149102 & GO:0032104 & $\begin{array}{l}\text { GO_REGULATION_OF_RESPONSE_TO_ } \\
\text { EXTRACELLULAR_STIMULUS }\end{array}$ & 0.00553166 & $1.85 \mathrm{E}-04$ \\
\hline \multicolumn{6}{|c|}{ 5. Differentiation ( $n=19$ GO-terms) } \\
\hline 1.16989444 & 87.1873536 & GO:0007569 & GO_AGING & 0.00620263 & $2.35 \mathrm{E}-04$ \\
\hline 1.29715264 & 64.7572209 & GO:0060348 & GO_BONE_DEVELOPMENT & 0.003857 & 6.89E-05 \\
\hline 1.28981373 & 55.8220141 & GO:0007571 & GO_CELL_AGING & 0.01100813 & $8.08 \mathrm{E}-04$ \\
\hline 1.30488668 & 55.1772053 & GO:0030901 & GO_MIDBRAIN_DEVELOPMENT & 0.00892851 & 5.29E-04 \\
\hline 1.37923729 & 44.952381 & GO:0060350 & GO_BONE_MORPHOGENESIS & 0.00804898 & 3.94E-04 \\
\hline \multicolumn{6}{|c|}{ 6. Immune system ( $n=11$ GO-terms) } \\
\hline 0.99092454 & 113.025761 & GO:0002683 & $\begin{array}{l}\text { GO_REGULATION_OF_IMMUNE_SYSTEM_ } \\
\text { PROCESS }\end{array}$ & 0.63535319 & 0.6209274 \\
\hline 1.10959897 & 93.7275566 & GO:0002253 & GO_IMMUNE_EFFECTOR_PROCESS & 0.02435747 & 0.00394916 \\
\hline 1.01653513 & 90.5035129 & GO:0002684 & $\begin{array}{l}\text { GO_NEGATIVE_REGULATION_OF_IMMUNE_ } \\
\text { SYSTEM_PROCESS }\end{array}$ & 0.37567449 & 0.33351316 \\
\hline 1.2647688 & 47.4395004 & GO:0002507 & GO_LYMPHOCYTE_MEDIATED_IMMUNITY & 0.03122183 & 0.00639526 \\
\hline 1.60515729 & 23.6736924 & GO:0001776 & $\begin{array}{l}\text { GO_MYELOID_DENDRITIC_CELL_ACTIVA- } \\
\text { TION }\end{array}$ & 0.00827038 & $4.71 \mathrm{E}-04$ \\
\hline \multicolumn{6}{|c|}{ 7. Immune system differentiation ( $n=9$ GO-terms) } \\
\hline 1.28373491 & 53.7494145 & GO:0002273 & GO_MYELOID_CELL_HOMEOSTASIS & 0.01435436 & 0.00146033 \\
\hline 1.70560294 & 21.6932084 & GO:0097029 & GO_DENDRITIC_CELL_DIFFERENTIATION & 0.00506811 & $1.55 \mathrm{E}-04$ \\
\hline 1.79932578 & 16.6729118 & GO:0043011 & $\begin{array}{l}\text { GO_MYELOID_DENDRITIC_CELL_ } \\
\text { DIFFERENTIATION }\end{array}$ & 0.0066832 & $2.68 \mathrm{E}-04$ \\
\hline 1.39259772 & 15.7978142 & GO:0045650 & $\begin{array}{l}\text { GO_REGULATION_OF_MACROPHAGE_ } \\
\text { DIFFERENTIATION }\end{array}$ & 0.08104498 & 0.03640847 \\
\hline 1.64144679 & 13.4028103 & GO:0045656 & $\begin{array}{l}\text { GO_REGULATION_OF_MONOCYTE_ } \\
\text { DIFFERENTIATION }\end{array}$ & 0.02937604 & 0.00562175 \\
\hline \multicolumn{6}{|c|}{ 8. Apoptosis ( $n=8 \mathrm{GO}$-terms) } \\
\hline 1.11228814 & 67.4285714 & GO:2001237 & $\begin{array}{l}\text { GO_REGULATION_OF_EXTRINSIC_ } \\
\text { APOPTOTIC_SIGNALING_PATHWAY }\end{array}$ & 0.11169862 & 0.06124028 \\
\hline 1.32504839 & 52.8282592 & GO:0097191 & $\begin{array}{l}\text { GO_EXTRINSIC_APOPTOTIC_SIGNALING_ } \\
\text { PATHWAY }\end{array}$ & 0.00816894 & 4.17E-04 \\
\hline 1.18811615 & 52.1834504 & GO:2001238 & $\begin{array}{l}\text { GO_NEGATIVE_REGULATION_OF_-̄_ } \\
\text { EXTRINSIC_APOPTOTIC_SIGNALING_ } \\
\text { PATHWAY }\end{array}$ & 0.06539505 & 0.0254644 \\
\hline 1.20186012 & 38.2740047 & GO:2001239 & $\begin{array}{l}\text { GO_POSITIVE_REGULATION_OF_- } \\
\text { EXTRINSIC_APOPTOTIC_SIGNALING_ } \\
\text { PATHWAY }\end{array}$ & 0.09885752 & 0.0504707 \\
\hline 1.71311452 & 26.8516784 & GO:0097192 & & 0.00255118 & $1.53 \mathrm{E}-05$ \\
\hline
\end{tabular}


Table 4 (Continued)

\begin{tabular}{|c|c|c|c|c|c|}
\hline Enrichment & $\begin{array}{l}\text { Expected } \\
\text { overlap }\end{array}$ & GO ID & Pathway & $q$-Value & p-Value \\
\hline & & & $\begin{array}{l}\text { GO_EXTRINSIC_APOPTOTIC_SIGNALING_ } \\
\text { PATHWAY_IN_ABSENCE_OF_LIGAND }\end{array}$ & & \\
\hline \multicolumn{6}{|c|}{ 9. Hormones ( $n=8$ GO-terms) } \\
\hline 1.48644638 & 41.0374707 & GO:0034959 & GO_SECRETORY_GRANULE_LUMEN & 0.00314492 & $3.83 \mathrm{E}-05$ \\
\hline 1.73873247 & 22.4301327 & GO:0034230 & $\begin{array}{l}\text { GO_CELLULAR_RESPONSE_TO_AMINO_ } \\
\text { ACID_STARVATION }\end{array}$ & 0.00376962 & $6.17 \mathrm{E}-05$ \\
\hline 1.97380586 & 12.665886 & GO:0016486 & GO_PEPTIDE_HORMONE_PROCESSING & 0.00561715 & $1.95 \mathrm{E}-04$ \\
\hline 1.97380586 & 11.6526151 & GO:0045929 & $\begin{array}{l}\text { GO_POSITIVE_REGULATION_OF_DNA_ } \\
\text { RECOMBINATION }\end{array}$ & 0.00768437 & $3.45 \mathrm{E}-04$ \\
\hline 1.69763549 & 11.1920375 & GO:0032346 & $\begin{array}{l}\text { GO_POSITIVE_REGULATION_OF_- } \\
\text { CHONDROCYTE_DIFFERENTIATION }\end{array}$ & 0.03119784 & 0.00637148 \\
\hline \multicolumn{6}{|c|}{ 10. Bone / digestive development ( $n=7$ GO-terms) } \\
\hline 1.45787099 & 38.412178 & GO:0070167 & $\begin{array}{l}\text { GO_REGULATION_OF_BIOMINERAL_ } \\
\text { TISSUE_DEVELOPMENT }\end{array}$ & 0.00562511 & $1.97 \mathrm{E}-04$ \\
\hline 1.87258504 & 19.7587822 & GO:0044058 & $\begin{array}{l}\text { GO_REGULATION_OF_DIGESTIVE_SYSTEM_ } \\
\text { PROCESS }\end{array}$ & 0.00256516 & $1.78 \mathrm{E}-05$ \\
\hline 2.09122773 & 16.2583919 & GO:0060451 & $\begin{array}{l}\text { GO_CARTILAGE_DEVELOPMENT_INVOLVE- } \\
\text { D_IN_ENDOCHONDRAL_BONE_- } \\
\text { MORPHOGENESIS }\end{array}$ & 0.00148804 & $3.32 \mathrm{E}-06$ \\
\hline 1.79272275 & 15.0608899 & GO:0046877 & $\begin{array}{l}\text { GO_PHOSPHATIDYLINOSITOL_ } \\
\text { DEPHOSPHORYLATION }\end{array}$ & 0.00919742 & $5.80 \mathrm{E}-04$ \\
\hline 2.10965484 & 11.3762685 & GO:0060457 & $\begin{array}{l}\text { GO_NEGATIVE_REGULATION_OF_ } \\
\text { DIGESTIVE_SYSTEM_PROCESS }\end{array}$ & 0.004262 & $8.71 \mathrm{E}-05$ \\
\hline
\end{tabular}

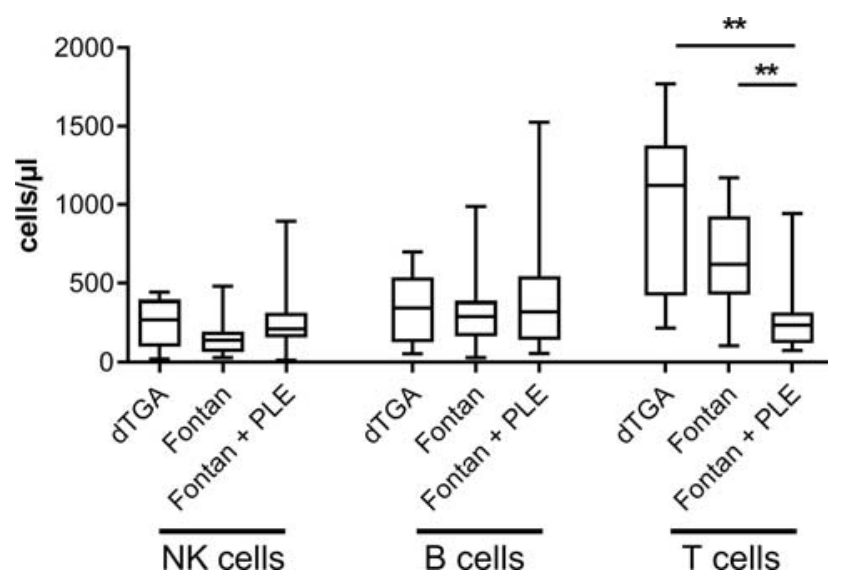

Fig. 3 Reduction in T cells in Fontan patients with protein-losing enteropathy (PLE). Absolute cell counts of natural killer (NK) cells, B cells and T cells of all dextro-transposition of the great arteries (dTCA) patients, Fontan patients with and without PLE. Box plots depict the 75th percentile, median and 25th percentile, values and whiskers represent maximum and minimum values. ${ }^{*} p<0.05,{ }^{* *} p<0.01,{ }^{* * *} p<0.001$.

differentiation of CD4+ and CD8 + and increased Treg frequency in Fontan patients with PLE. Both can be linked to significant changes in immunological pathways including leucocyte mediated immunity and migration in Fontan patients with PLE.

The characterization of a global picture of miRNA target networks helps to understand biological processes involved in disease development. As there is increasing evidence that PLE is associated with lymphatic dysfunction in Fontan
A
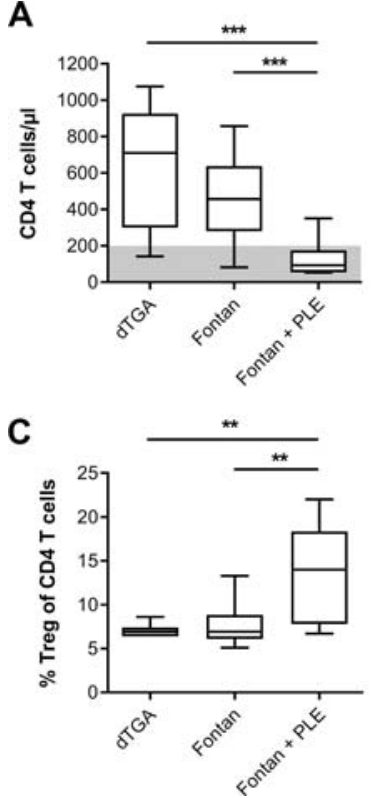

B

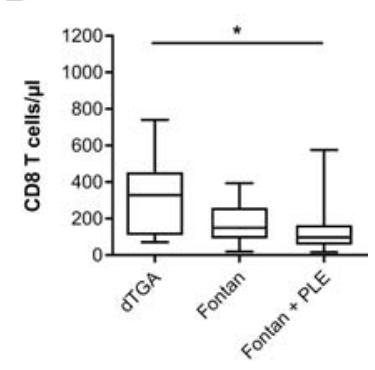

D

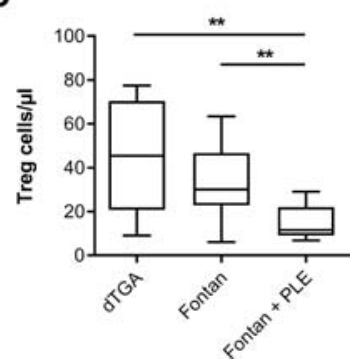

Fig. 4 Reduction in CD4+ T lymphocytes in Fontan patients with protein-losing enteropathy (PLE). Absolute numbers of CD4+ T cells (A) and CD8+ T cells (B) were determined in dextro-transposition of the great arteries (dTGA) patients, Fontan patients with and without PLE. (C) Percentage of Treg cells (CD4 +/CD25 +/CD127-) among all CD4+ T cells. (D) Absolute cell count of Treg cells. Box plots depict the 75th percentile, median and 25th percentile, values and whiskers represent maximum and minimum values. ${ }^{*} p<0.05,{ }^{* *} p<0.01$, ${ }^{* * *} p<0.001$. 
e18 Lymphocyte Immune Response and T Cell Differentiation in Fontan Patients with PLE Moosmann et al.

A1

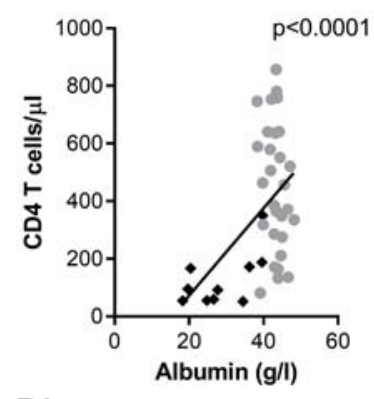

B1

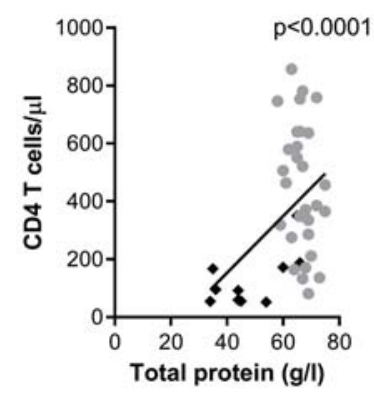

C1

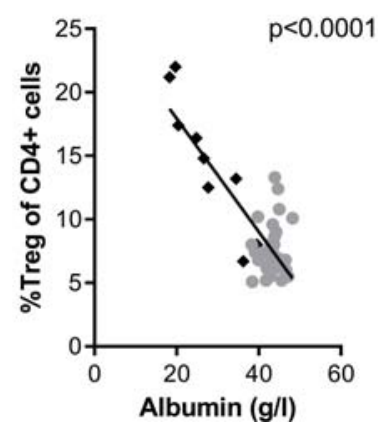

A2

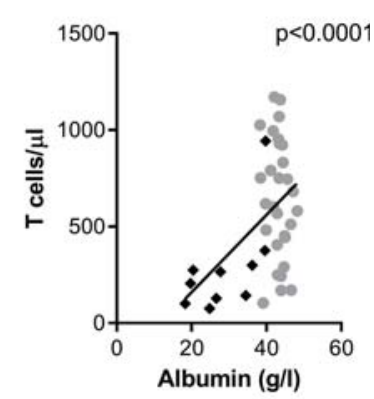

B2

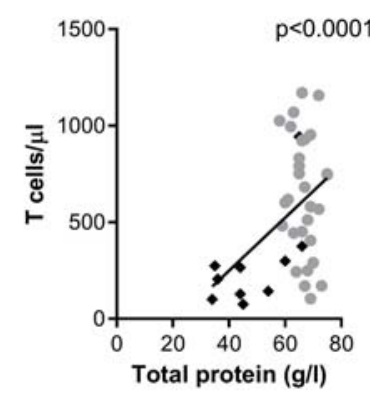

C2

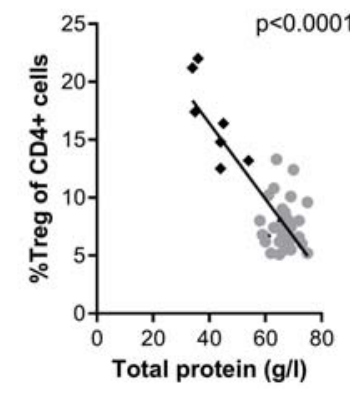

Fig. 5 Correlations of $\mathrm{T}$ cell count and subsets with routine laboratory analysis. (A1) CD4+ cell count and albumin (A2) T cell count and albumin (B1) CD4+ cell count and total protein (B2) T cell count and total protein (C1) Treg frequency and albumin (C2) Treg frequency and total protein. Black rectangles representing Fontan patients with protein-losing enteropathy (PLE), black circles Fontan patients without PLE. Correlations were calculated using nonparametric Spearman's rank correlation coefficient.

patients, the in silico pathway analysis facilitated identification of miRNA regulated target networks highlighting the involvement of lymphatic and immunologic disease pathways in Fontan patients with PLE.

Ten significantly changed networks, thereof five associated with immune response including leukocyte-mediated immunity, immune system development, activation of immune response, leukocyte migration, and monocyte differentiation were identified. In addition, networks involving pathways of the gastrointestinal tract, which is clinically significantly affected in patients with PLE, were identified as severely altered. Digestive symptoms including diarrhea and malnutrition represent one of the main clinical symptoms of PLE.

Furthermore, we identified significantly different expressed miRNAs when comparing Fontan with PLE to Fontan without PLE and dTGA. miR-150-5p could be identified as the most

downregulated miRNA. miR-150-5p is expressed in lymph nodes, spleen, and mature immune cells ( $B$ and $T$ cells) and is an important regulator cell differentiation and activation of immune cells. ${ }^{15}$ Interestingly, miR-150-5p has been identified as a major regulator of the AKT3/BIM pathway controlling CD4 + and CD8 + T cell development, proliferation, and function. ${ }^{15}$ Moreover, this miRNA has been reported to negatively regulate formation and homeostatic turnover of memory CD8+ T cells and to promote increased expression of $\mathrm{K}_{\mathrm{i}}-67$ suggesting an enhanced ongoing proliferation. ${ }^{16}$ Downregulation of miR150-5p has been associated with poorly cell differentiation and decreased outcome for cancer patients. As miR-150 is significantly downregulated in Fontan patients with PLE, this mechanism might play a role in PLE development as we observed pronounced alterations in $\mathrm{T}$ cell differentiation.

A decrease in lymphocyte and CD4+ count in Fontan patients with PLE has already been described. ${ }^{5,17,18}$ We demonstrate that the decline of T cells in Fontan patients with PLE is due to both, a reduction in CD4+ T helper and CD8 + cytotoxic T cells. However, the depletion of T cells is mainly dependent on the reduction in CD4 $+\mathrm{T}$ helper cells. Low CD4+ cell count results in a remarkable change in $\mathrm{CD} 4+/ \mathrm{CD} 8+$ ratio postponed to $1: 1$. A low $\mathrm{CD} 4+\mathrm{T}$ cell count below $<200$ cells $/ \mu \mathrm{L}$ is formally defined as AIDS-defining illness according to the guidelines of Centers for Disease Control and Prevention (CDC), regardless of the presence of symptoms or opportunistic diseases. ${ }^{19}$ Of importance, 9 out of 10 Fontan patients with PLE and 5 out of 30 Fontan patients without PLE showed a CD $4+$ T cell count $<200$ cells $/ \mu L$. These five Fontan patients without PLE did not show clinical symptoms of PLE according to our definition; however, as we already know from other clinical studies, these patients might already have lymphatic dysregulation, potentially worsening in the future. Due to the substantial T cell deficiency in Fontan patients with PLE, we characterized CD4+ and CD8+ differentiation pattern in all patients. The ability of T cells to differentiate from naïve to memory and effector subsets represents a critical determinant of host protection against pathogens and autoimmunity. ${ }^{20}$ We identified a nearly complete loss of naïve CD4+ and CD8 + cells and a shift from the naïve T cell pool to memory and terminal differentiate T cells (TEMRA) in Fontan patients with PLE. We have not seen any of these changes in Fontan patients without PLE or biventricular ITGA patients. The changes observed in CD4+ and CD8 + cell differentiation might suggest an ongoing immune reaction in Fontan patients with PLE, where naïve $T$ cells rapidly differentiate to memory or TEMRA cells after antigen recognition. ${ }^{20,21}$ However, activation of $\mathrm{T}$ cells also results in proliferation, clonal expansion, and accumulation of memory and/or effector cells. In contrast, we found a decrease in absolute CD4 + cell counts in all differentiation stages suggesting that inappropriate $\mathrm{T}$ cell regeneration, survival, or loss (due to enteropathy including reduced intestinal mucosal immune function or abnormal lymphatic pattern) induces an aberrant immune phenotype rather than a "normal" immune reaction. $^{18}$

To further investigate the clinical relevance of $\mathrm{CD} 4+$ deficiency, subpopulations of the remaining CD4+ T cells in Fontan patients with PLE were analyzed. Treg cells are functionally distinct subset of mature CD4 + T cells with high 
A

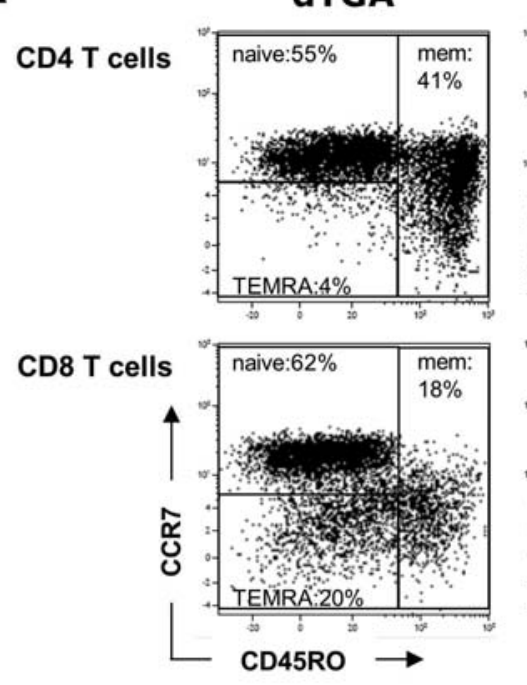

Fontan
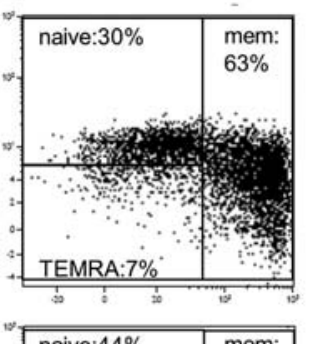

Fontan + PLE
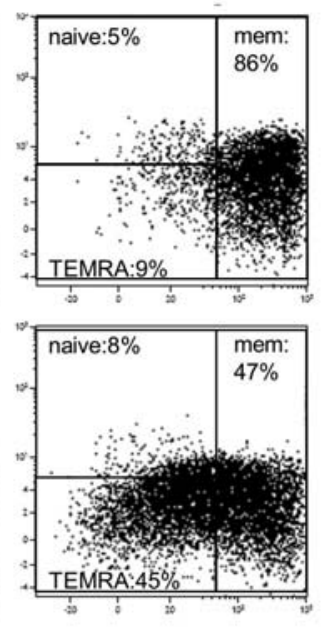

B

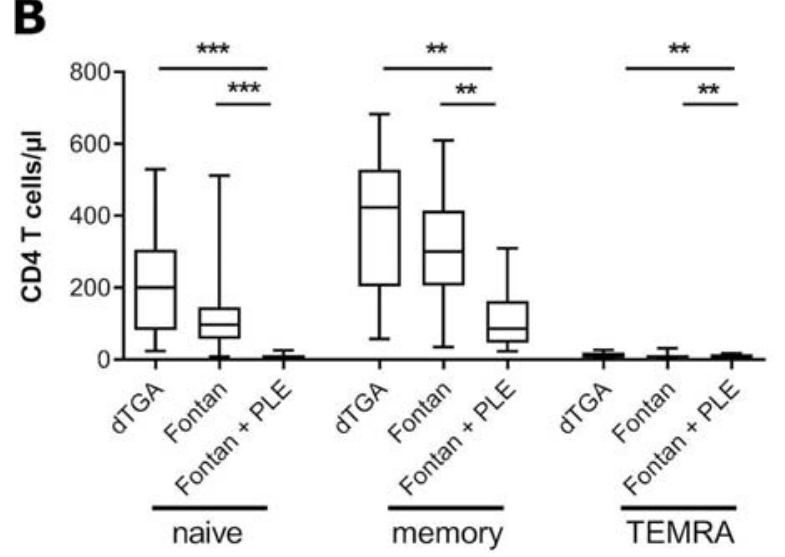

C

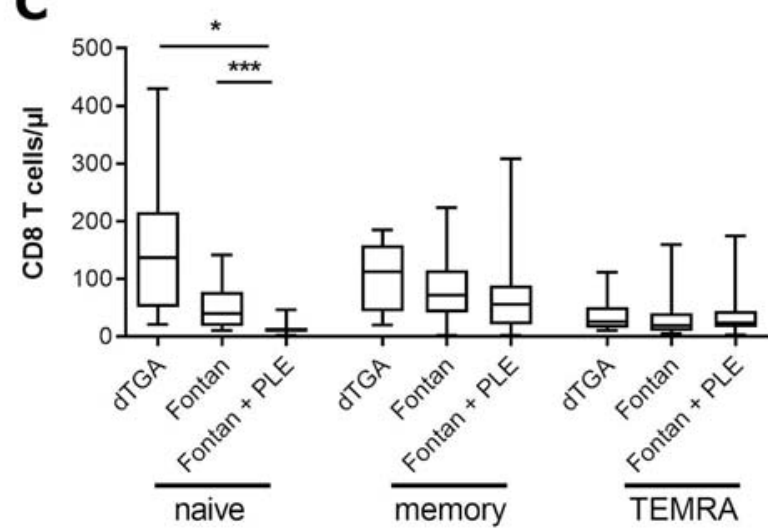

Fig. 6 Loss of naïve T cell compartment in Fontan patients with protein-losing enteropathy (PLE). (A) CD4+ T cells and CD8+T cells from dextrotransposition of the great arteries (dTGA), Fontan with and without PLE were analyzed for CCR7 and CD45RO expression. Plots show representative data: naïve (CCR7 +/CD45RO-), memory (CD45RO +), and TEMRA (CCR7-/CD45RO-) cells. (B, C). Box plots depict the 75th percentile, median and 25 th percentile, values and whiskers represent maximum and minimum values. ${ }^{*} p<0.05,{ }^{* *} p<0.01,{ }^{* * *} p<0.001$.

immune suppressive activity. Treg cells play a key role in the maintenance of self-tolerance and homeostasis, preventing autoimmune diseases, and limiting chronic inflammation. ${ }^{22}$ The absolute count of Treg cells is decreased in Fontan patients with PLE, due to the pronounced reduction in total CD4+ cells. However, the frequency of CD4+ Treg cells shows a twofold increase in Fontan patients with PLE, suggesting that PLE is associated with loss of specific subsets within the CD4+ T cell compartment. The increase in Treg cell frequency could be induced by chronic inflammation, but may lead to an unsuccessful immune response. The severity of PLE can be clinically addressed by the degree of hypoalbuminemia and low total protein, both showed significant correlation to T cell numbers and subsets. Oral steroids are a common treatment strategy for patients with severe symptoms of PLE. High-dose steroids can acutely affect differentiation of T cell subsets, especially of CD4+ T cells and naïve T cells. Therefore, we raise the question whether steroid therapy would additionally affect differentiation and cell count unfavorably. Based on these data, other immunomodulatory treatment strategies could play a role in the treatment of PLE in the future. However, not only the absolute cell numbers but also the functional capacity of the remaining $\mathrm{T}$ cells to participate in the immune response needs to be investigated to answer that question.

Clinically, magnetic resonance imaging studies in Fontan patients describe the morphology of lymphatic malformations after the Fontan procedure. ${ }^{4,14}$ As we did not perform simultaneous T2-weighted lymphatic imaging in all patients, we suggest that additional studies are needed to illustrate the interplay between lymphatic malformations and $\mathrm{T}$ cell response. Further, we have no information about the timely correlation of lymphatic flow alterations, T cell alteration, and functional immunological responses in Fontan patients in the course of Glenn and Fontan surgery and the time of occurrence of PLE symptoms, which needs further evaluation in this context.

\section{Limitations}

The investigated sample size of the biventricular dTGA group was relatively small. However, we wanted to include this 
group as a comparison group, as they also had neonatal cardiac surgery, to avoid bias on lymphopenia and $\mathrm{T}$ cell count due to early thymectomy. Potential influencing factors such as age at the time of the study and gender were not included in the analysis. We did not include simultaneous T2-weighted lymphatic imaging to allow further statements about the correlation between lymphatic congestion/abnormalities and $\mathrm{T}$ cell response.

\section{Conclusion}

PLE in Fontan patients is associated with severe lymphopenia, $\mathrm{T}$ cell deficiency, significant alterations of T cell differentiation, and increased Treg frequency reflecting an immune status of chronic inflammation and shortened protection against pathogens and autoimmunity. These cellular alterations seem to be dysregulated by several miRNA controlled immunological pathways. The interplay between lymphatic circulation and cellular lymphatic dysfunction in the progress of PLE development needs further evaluation.

Conflict of Interest

The authors have no conflicts of interest to declare.

\section{Acknowledgments}

This work was supported by the DGPK and the German Heart Foundation through the Gerd-Killian grant. We thank Florentine Koppitz (Core Unit Cell Sorting and Immunomonitoring Erlangen) for FACS analysis.

Simon Völkl and Sven Dittrich contributed equally to this manuscript, as this was a translational work between human genetics, our immunomonitoring unit, and the Department of Pediatric Cardiology.

\section{References}

1 Khairy P, Fernandes SM, Mayer JE Jr, et al. Long-term survival, modes of death, and predictors of mortality in patients with Fontan surgery. Circulation 2008;117(01):85-92

2 Rychik J. Protein-losing enteropathy after Fontan operation. Congenit Heart Dis 2007;2(05):288-300

3 Ghosh RM, Griffis HM, Glatz AC, et al. Prevalence and cause of early Fontan complications: does the lymphatic circulation play a role? J Am Heart Assoc 2020;9(07):e015318

4 Mohanakumar S, Telinius N, Kelly B, et al. Morphology and function of the lymphatic vasculature in patients with a Fontan circulation. Circ Cardiovasc Imaging 2019;12(04):e008074

5 Kovacikova L, Krasnanova V, Skrak P, et al. Immune abnormalities in patients with single ventricle circulation precede the Fontan procedure. World J Pediatr Congenit Heart Surg 2017;8(06): 672-682
6 Horvitz HR, Sulston JE. Isolation and genetic characterization of cell-lineage mutants of the nematode Caenorhabditis elegans. Genetics 1980;96(02):435-454

7 Bartel DP. MicroRNAs: genomics, biogenesis, mechanism, and function. Cell 2004;116(02):281-297

8 Goren Y, Kushnir M, Zafrir B, Tabak S, Lewis BS, Amir O. Serum levels of microRNAs in patients with heart failure. Eur J Heart Fail 2012;14(02):147-154

9 Satoh J, Tabunoki H. Comprehensive analysis of human microRNA target networks. BioData Min 2011;4:17

10 Halnon NJ, Jamieson B, Plunkett M, Kitchen CM, Pham T, Krogstad $P$. Thymic function and impaired maintenance of peripheral $\mathrm{T}$ cell populations in children with congenital heart disease and surgical thymectomy. Pediatr Res 2005;57(01):42-48

11 Rychik J, Atz AM, Celermajer DS, et al;American Heart Association Council on Cardiovascular Disease in the Young and Council on Cardiovascular and Stroke Nursing. Evaluation and management of the child and adult with Fontan circulation: a scientific statement from the American Heart Association. Circulation

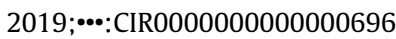

12 Chou CH, Chang NW, Shrestha S, et al. miRTarBase 2016: updates to the experimentally validated miRNA-target interactions database. Nucleic Acids Res 2016;44(D1):D239-D247

13 Godard P, van Eyll J. Pathway analysis from lists of microRNAs: common pitfalls and alternative strategy. Nucleic Acids Res 2015; 43(07):3490-3497

14 Biko DM, DeWitt AG, Pinto EM, et al. MRI evaluation of lymphatic abnormalities in the neck and thorax after Fontan surgery: relationship with outcome. Radiology 2019;291(03):774-780

15 Ghisi M, Corradin A, Basso K, et al. Modulation of microRNA expression in human T-cell development: targeting of NOTCH3 by miR-150. Blood 2011;117(26):7053-7062

16 Sang W, Sun C, Zhang C, et al. MicroRNA-150 negatively regulates the function of $\mathrm{CD} 4(+) \mathrm{T}$ cells through AKT3/Bim signaling pathway. Cell Immunol 2016;306-307:35-40

17 Magdo HS, Stillwell TL, Greenhawt MJ, et al. Immune abnormalities in Fontan protein-losing enteropathy: a case-control study. J Pediatr 2015;167(02):331-337

18 Lenz D, Hambsch J, Schneider P, Tárnok A. Protein-losing enteropathy after Fontan surgery: is assessment of risk patients with immunological data possible? Cytometry B Clin Cytom 2003;53(01):34-39

19 Schneider E, Whitmore S, Glynn KM, Dominguez K, Mitsch A, McKenna MTCenters for Disease Control and Prevention (CDC) Revised surveillance case definitions for HIV infection among adults, adolescents, and children aged $<18$ months and for HIV infection and AIDS among children aged 18 months to $<13$ years-United States, 2008. MMWR Recomm Rep 2008;57 (RR-10):1-12

20 Larbi A, Fulop T. From "truly naïve" to "exhausted senescent" T cells: when markers predict functionality. Cytometry A 2014;85 (01):25-35

21 Pepper M, Jenkins MK. Origins of CD4(+) effector and central memory T cells. Nat Immunol 2011;12(06):467-471

22 Hardy MY, Vari F, Rossetti T, Hart DN, Prue RL. A flow cytometry based assay for the enumeration of regulatory T cells in whole blood. J Immunol Methods 2013;390(1-2):121-126 